\title{
Non-Radial Pulsations of Distorted Stellar Structures: Effect of Mass Variation
}

\author{
Seema Saini \\ Department of Mathematics, \\ Graphic Era Deemed to be University, Dehradun, Uttarakhand, India. \\ Corresponding author: sainiseema1@gmail.com \\ Sunil Kumar \\ Department of Mathematics, \\ Graphic Era Hill University, Dehradun, Uttarakhand, India. \\ E-mail: dr.sunilkumar@live.com \\ Vineet Bhatt \\ Department of Mathematics, \\ Chandigarh University, Mohali, Punjab, India. \\ E-mail: vineet.bhatt58@gmail.com \\ Pradeep Bedi \\ Department of Computer Science and Engineering, \\ Lingaya's Vidyapeeth, Faridabad, Haryana, India. \\ E-mail: bedipradeep1983@gmail.com
}

(Received on April 24, 2021; Accepted on September 18, 2021)

\begin{abstract}
Eigen-frequencies (EF) of non-radial modes (NRM) of pulsations of differentially rotating (D R) and tidally distorted (T D) stellar models by considering the effect of mass variation (MV) on its equi-potentials surfaces inside a star. The method utilizes an averaging proposal of Kippenhahn and Thomas ( $\mathrm{K}$ and $\mathrm{T}$ ) with conjunction of the concept of Roche-equipotential. The study accolades and corrects earlier studies of non-radial (NR) pulsations of DR and TD stellar structures of different natures such as radial and non-radial oscillations, X-ray, gamma ray and other electromagnetic disturbances. The reflection of the work comes from the requirements of the inclusion of nonuniform densities that yield Lane-Emden equation to have reliable results up to second order disturbances.
\end{abstract}

Keywords- Equipotential surface, Non- radial oscillations, Differential rotation, Tidal distortions, Polytropic models, Mass variation.

\section{Introduction}

Observations have shown that pulsars are suffered by different types of mechanical perversions, such as differential rotation, vibrations, non-uniform densities inside, along their lifetime in order to reproduce $\mathrm{X}$ ray, gamma-ray, different types of waves and other electromagnetic perturbations Glendenning (2012). The analysis of oscillations spectrum provides an unrivaled method for probing the stellar structure (internal) because the frequencies of these pulsations depends upon the sound speed inside the star, which in turn depends on the density, temperature, gas motion and other properties of stellar interior. Analytic study of the problem of determining the equilibrium structures, periods of pulsations and stability of rotationally and tidally (RT) distorted stellar models is quite complex. It will become more complex, if the rotation is differential. 
This work is associated with the pulsation periods of stellar models of NR oscillations, affected by mass variation happening at various stages of stars. Most of the authors such as, Mohan et al. (1991) formulated EF of NRM of pulsations of rotationally and tidally (R and T) distorted stellar models. There are several reasons which are responsible for these oscillations, such as, collaborating a binary or multiple systems, impact of the inter-stellar medium, glitches and other electromagnetic perturbations Glendenning (2012). Chandrasekhar (1964) has given this approach in his work. However, it was applied initially only to polytropic equation of state, and some years later to more realistic pulsars. Several authors such as Yojisoki (1986), Chandrasekhar and Ferrari (1991) have made significant contributions to the studies of the problems of NR pulsations of stars. Chandrasekhar and Ferrari (1991) have proposed a complete theory of NRM oscillations of a static spherical symmetric distribution of matter described in terms of energy density and isotropic pressure on the premise that oscillations are excited by incident gravitational waves. Telting and Schrijvers (1997) studied NR adiabatic pulsations for some models of a rotating stellar model and generated time series of absorption line profiles. Clement (1967) also discussed normal modes of oscillations for rotating stars using a new numerical method for computing non-radial eigenfunctions. The approach of calculating normal modes of spherical stellar models is generalized to two dimensions. While calculating EF of distorted modes some parameters such as, Coriolis force, electromagnetic disturbances are ignored. However, for most of the models, a better approximation to the density distribution of a star can be achieved by MV from centre to surface of the star. Therefore, it appears quite possible to obtain some conclusions regarding the effects of density distribution on the pulsation properties of (D R) and (T D) polytropic stars of different polytropic indices and with interfaces of different radii. Pressure play an important role to calculate pulsations hence, many authors such as Brown (1984), Lovekin \& Deupree (2008), Petrovay et al. (2007), Schlattl et al. (1997). Pulsation in NRM causes deviation from spherical symmetry, the star changes its shape and size. Recently, De Ridder et al. (2009), discussed long lifetimes in giant stars of non-radial stars these effects have been carried out by Ball et al. (2016), Trampedach et al. (2017). It has been observed that pressure $(\mathrm{P})$ modes of gaseous sphere are highly sensitive at the exterior.

The technique presented to study linear adiabatic non-radial pulsations, leads authors for obtaining an apparent scheme to classify pulsations modes. The suggested approach only depends on the boundary conditions (BC) at endpoints (centre and outer surface) of stellar interior. This classification is independent to the number of nodes of eigen-functions used to describe oscillatory motion. However, the sequence of EF can gives the sufficient information of the oscillatory system. The system of equations for the motion of linear adiabatic NRM of stellar pulsations has been reduced to a set of second order differential equation, and sufficient to obtain a more reliable classification scheme of stellar pulsations. For isolated compact spheres, NRM of oscillations are generally occurs due to density and pressure disturbances. In order to distinguish, fundamental modes ( $f$ modes), pressure modes ( $p$ modes) and gravity modes, ( $g$ modes) respectively. Flores \& Lugones (2010) correlated strange, hadronic and hybrid stars, with them. These $f, p, g$ modes play a vital role in analyzing the corresponding pulsations of gravitational emission hence, it is required to inspect the corresponding pulsation frequencies. According to the theory of Flores \& Lugones (2010), it is very tough to detect hadronic stars of mass range 1.4 2.0 M from strange stars for the case of ( $f$ modes, whereas it is observed that there is a broad variety of determination in the $\mathrm{EF}$ of the neutron stars in case of modes $p$ modes, while $g$ modes arises due to the sharp density discontinuities in the pulsar. Kumar et al. (2015) have 
calculated NRM of oscillations to determine vibrational stabilities of (D R) polytropic stars. Saini et al. (2015) have also been discussed radial oscillations of (D R) and (T D) polytropic stars.

In the present paper we extend the approach of Mohan et al. (1991) in corporation with the averaging approach of Kippenhahn and Thomas (1970) with conjunction of the concept of Roche-equipotentials. Mohan et al. (1991) developed the approach to study stellar structures and their pulsations behaviors, but, this approach was limited for stellar models of uniform densities. However, in case of realistic stars, densities vary from centre to surface due to various physical effects such as gravity, tides, and rotations etc. Therefore, it is necessary to modify the existing approach for drawing more accurate results related to the structures and oscillations of realistic stars.

This article consists of six sections. Section 1 is the Introduction. EF of pseudo NRM of oscillation has been discussed in section 2. This approach is then used in section 3 to compute EF of NRM of oscillations for polytropic models of gaseous spheres. Computational results for the EF of NRM are discussed in section 4. Result and discussion are dealt in section 5 whereas conclusion and scope for future studies are discussed in section 6.

Table 1. Representations of symbols.

\begin{tabular}{|l|l|}
\hline Symbol & Notation \\
\hline$\varsigma, \eta, \phi$ & Non dimensional eigenfunctions \\
\hline$P_{\psi}, \rho_{\psi}$ & Pressure and density on equi-potentials surfaces \\
\hline$\psi$ & Total potential \\
\hline$\sigma$ & (EF of pulsations) \\
\hline$l$ & AQN(azimuthal quantum number) \\
\hline$G$ & gravitational constant \\
\hline$\rho_{c}$ & density at centre \\
\hline$\delta r_{\psi}$ & Amplitudes of mean of the Lagrangian variation \\
\hline$P_{\psi}^{\prime}, \psi_{g}^{\prime}$ & pressure and gravitational amplitudes \\
\hline $\mathrm{R}$ & Partition between the centre of masses \\
\hline$q=\frac{M_{1}}{M_{0}}$ & Ratio of mass (by which primary component is being distorted tidally) \\
\hline$M_{0}(r)$ & Mass interior of a spherical structure of radius r \\
\hline
\end{tabular}

\section{Computation of EF of Small Adiabatic NRM}

A distorted model of (D R) and (T D) gaseous spheres may be used to analyze EF of NRM of pulsations. Mohan and Saxena (1985), Chanderaskhar and Ferrari (1991) have studied the pulsations of stars. In this study it is assumed that models rotate as a solid body with uniform mass distribution. Mohan et al. (1991) determine the EF of radial and NRM of oscillations for R and T distorted gaseous spheres. Later this approach was used by Mohan et al. (1991), Lal (1993) to calculate EF of differentially rotating (D R) stars. In general, the approach adopted by Kippenhahn and Thomas (1970) can also be used to establish the eigen-value problem in a manner that it could determines EF of small radial and NRM of pulsations of differentially rotating stars. Using the generalized approach of Kippenhahn and Thomas (1970) and Mohan et al. (1991) the EF of NRM of pulsations of (D R) and (T D) spheres using the law:

$\omega^{2}=b_{1}+b_{2} s^{2}+b_{3} s^{4}$ 
Here, $\omega$ is a angular velocity and $b_{1}, b_{2}$ are constants.

$$
\begin{aligned}
& \frac{d \zeta}{d x}+B_{1}^{*} \zeta+\left(B_{2}^{*}+\frac{1}{\sigma^{2}} B_{3}^{*}\right) \eta+\frac{1}{\sigma^{2}} B_{3}^{*} \phi=0, \\
& \frac{d \eta}{d x}+\left(E_{1}^{*} \sigma^{2}+E_{2}^{*}\right) \zeta+E_{3}^{*} \eta+E_{4}^{*} \phi+\frac{d \phi}{d x}=0,
\end{aligned}
$$

and

$$
\frac{\mathrm{d}^{2} \phi}{d x^{2}}+F_{1}^{*} \frac{d \phi}{d x}+F_{2}^{*} \zeta+F_{3}^{*} \eta+F_{4}^{*} \phi=0
$$

where,

$$
\begin{aligned}
& B_{1}^{*}=\frac{l+1}{x}+\frac{1}{\gamma P_{\psi}} \frac{d P \psi}{d x}, \\
& B_{2}{ }^{*}=\frac{2 \pi G \rho_{c}}{R x}\left(\rho_{\psi} / \gamma P_{\psi}\right) r_{\psi}^{2} d r_{\psi} / d x \\
& =\frac{2 \pi R^{2} G \rho_{c}}{\gamma P_{\psi}} \rho_{\psi} r_{0 s}{ }^{2} x\left[\begin{array}{l}
1+\frac{2 b_{1}{ }^{2} r_{o s}{ }^{3} x^{3}}{z}+\frac{32 b_{1} b_{2} r_{0 s}{ }^{5} x^{5}}{15 z}+\left(\frac{36 q^{2}}{5 z^{2}}+\frac{12 b_{1}^{2} q}{5 z^{2}}\right) x^{6} r_{0 s}^{6}+ \\
+\frac{16}{21} \frac{b_{2}{ }^{2} r_{0 s}{ }^{7} x^{7}}{z}+\left(\frac{55 q^{2}}{7 z^{2}}+\frac{8 b_{1} b_{2} q}{21 z^{2}}\right) x^{8} r_{0 s}^{8} \\
+\left(\frac{26 q^{2}}{3 z^{2}}+\frac{124 b_{1} b_{2} q}{35 z^{2}}\right) x^{10} r_{0 s}{ }^{10}+\ldots
\end{array}\right], \\
& B_{3}^{*}=-\frac{l(l+1)}{R x} \frac{d r_{\psi}}{d x} 2 \pi G \rho_{c}, \\
& =-\frac{l(l+1)}{R x} 2 \pi G \rho_{c} r_{o s}\left[1+\frac{4 b_{1}^{2} r_{o s}^{3} x^{3}}{3 z}+\frac{8 b_{1} b_{2} r_{o s}^{5} x^{5}}{5 z}+\left(\frac{28 q^{2}}{5 z^{2}}+\frac{28 b_{1}^{2} q}{15 z^{2}}\right) r_{o s}^{6}+\right. \\
& \left.+\frac{64 b_{2}^{2} r_{o s}^{7} x^{7}}{105 z}+\left(\frac{45}{7 z^{2}} q^{2}+\frac{24 b_{1} b_{2} q}{7 z^{2}}\right) r_{o s}^{8} x^{8}+\left(\frac{22 q^{2}}{3 z^{2}}+\frac{22 b_{1} b_{2} q}{35 z^{2}}\right) r_{o s}^{10} x^{10}+\ldots\right] \\
& E_{1}^{*}=-\frac{1}{2 \pi G \rho_{c}} \frac{R x}{r_{\psi}^{2}} \frac{d r_{\psi}}{d x}, \\
& =-\frac{1}{2 \pi G \rho_{c} r_{0 s} x}\left[1+\frac{2 b_{1}{ }^{2} x^{3} r_{o s}{ }^{3}}{3 z}+\frac{16 b_{1} b_{2} x^{5} r_{o s}{ }^{5}}{15 z}+\left(\frac{4 q^{2}}{z^{2}}+\frac{4 b_{1}{ }^{2} q}{3 z^{2}}\right) x^{6} r_{o s}{ }^{6}+\right. \\
& \left.\frac{16 b_{2}{ }^{2} x^{7} r_{o s}{ }^{7}}{35 z}+\left(\frac{5 q^{2}}{z^{2}}+\frac{8 b_{1} b_{2} q}{3 z^{2}}\right) x^{8} r_{o s}{ }^{8}+\left(\frac{6 q^{2}}{z^{2}}+\frac{18 b_{1} b_{2} q}{35 z^{2}}\right) x^{10} r_{o s}{ }^{10}+\ldots\right] \text {, }
\end{aligned}
$$




$$
\begin{aligned}
& E_{2}^{*}=\frac{1}{2 \pi G \rho_{c}} \frac{A_{\psi}}{\rho_{\psi}} \frac{d P_{\psi}}{d x} \frac{R x}{r_{\psi}^{2}}, \\
& =\frac{1}{2 \pi G \rho_{c} D^{2}} \frac{1}{\rho_{\psi}}\left(\frac{1}{\rho_{\psi}} \frac{d \rho_{\psi}}{d x}-\frac{1}{\gamma P_{\psi}} \frac{d P_{\psi}}{d x}\right) \frac{d P_{\psi}}{d x} \frac{1}{x r_{o s}^{2}}\left[1-\frac{2 b_{1}{ }^{2} x^{3} r_{o s}{ }^{3}}{z}-\frac{32 b_{1} b_{2} x^{5} r_{o s}{ }^{5}}{15 z}-\right. \\
& -\left(\frac{36 q^{2}}{5 z^{2}}+\frac{12 b_{1}^{2} q}{5 z^{2}}\right) x^{6} r_{o s}{ }^{6}-\frac{16}{21 z} b_{2}{ }^{2} x^{7} r_{o s}{ }^{7}-\left(\frac{55 q^{2}}{7 z^{2}}+\frac{88 b_{1} b_{2} q}{21 z^{2}}\right) x^{8} r_{o s}{ }^{8}- \\
& \left.-\left(\frac{26 q^{2}}{3 z^{2}}+\frac{26 b_{1} b_{2} q}{35 z^{2}}\right) x^{10} r_{o s}{ }^{10}+\ldots\right], \\
& E_{3}^{*}=\frac{l}{x}+\left(\frac{1}{\rho_{\psi}} \frac{d \rho_{\psi}}{d x}-\frac{1}{\gamma P_{\psi}} \frac{d P_{\psi}}{d x}\right), E_{4}{ }^{*}=\frac{l}{x}, \\
& F_{1}^{*}=2 l / x-\frac{d^{2} r_{\psi} / d x^{2}}{d r_{\psi} / d x}+\frac{2}{r_{\psi}} \frac{d r_{\psi}}{d x}, \\
& =2(l+1) / x-\frac{1}{x}\left[\frac{2 b_{1}{ }^{2} x^{3} r_{o s}{ }^{3}}{z}+\frac{16 b_{1} b_{2} x^{5} r_{o s}{ }^{5}}{3 z}+\left(\frac{24 q^{2}}{z^{2}}+\frac{8 b_{1}{ }^{2} q}{z^{2}}\right) x^{6} r_{o s}{ }^{6}+\right. \\
& +\frac{112 b_{2}{ }^{2} x^{7} r_{o s}{ }^{7}}{35 z}+\left(\frac{40 q^{2}}{z^{2}}+\frac{448 b_{1} b_{2} q}{21 z^{2}}\right) x^{8} r_{o s}{ }^{8} \\
& \left.+\left(\frac{60 q^{2}}{z^{2}}+\frac{36 b_{1} b_{2} q}{7 z^{2}}\right) x^{10} r_{o s}{ }^{10}+\ldots\right] \\
& F_{2}^{*}=2 \frac{\rho_{\psi} x R}{\rho_{c} r_{\Psi}^{2}}\left(\frac{1}{\rho_{\Psi}} \frac{d \rho_{\Psi}}{d x}-\frac{1}{\gamma P_{\psi}} \frac{d P_{\psi}}{d x}\right)\left(\frac{d r_{\psi}}{d x}\right)^{2}, \\
& =2 \frac{\rho_{\psi}}{\rho_{c}}\left(\frac{1}{\rho_{\psi}} \frac{d P_{\psi}}{d x}-\frac{1}{\gamma P_{\psi}} \frac{d P_{\psi}}{d x}\right) \frac{1}{x r_{o s}}\left[1+\frac{2 b_{1}{ }^{2} x^{3} r_{o s}{ }^{3}}{3 z}+\frac{16 b_{1} b_{2} x^{5} r_{o s}{ }^{5}}{15 z}+\left(\frac{4 q^{2}}{z^{2}}+\frac{4 b_{1}{ }^{2} q}{3 z^{2}}\right) x^{6} r_{o s}^{6}\right. \\
& \left.+\frac{16 b_{2}{ }^{2} x^{7} r_{o s}{ }^{7}}{35 z}+\left(\frac{5 q^{2}}{z^{2}}+\frac{8 b_{1} b_{2} q}{3 z^{2}}\right) x^{8} r_{o s}{ }^{8}+\left(\frac{6 q^{2}}{z^{2}}+\frac{18 b_{1} b_{2} q}{35 z^{2}}\right) x^{10} r_{o s}{ }^{10}+\ldots\right] \text {, } \\
& F_{3}^{*}=-\frac{4 \pi G \rho_{\psi}^{2}}{\gamma P_{\psi}}\left(\frac{d r_{\psi}}{d x}\right)^{2}, \\
& =\frac{4 \pi G \rho_{\psi}^{2}}{\gamma P_{\psi}} r_{o s}^{2} R^{2}\left[1+\frac{8 b_{1}^{2} x^{3} r_{o s}{ }^{3}}{3 z}+\frac{16 b_{1} b_{2} x^{5} r_{o s}{ }^{5}}{5 z}+\left(\frac{56 q^{2}}{5 z^{2}}+\frac{56 b_{1}^{2} q}{15 z^{2}}\right) x^{6} r_{o s}^{6}+\right. \\
& \left.+\frac{128 b_{2}{ }^{2} x^{7} r_{0 s}{ }^{7}}{105 z}+\left(\frac{90 q^{2}}{7 z^{2}}+\frac{48 b_{1} b_{2} q}{7 z^{2}}\right) x^{8} r_{o s}{ }^{8}+\left(\frac{44 q^{2}}{3 z^{2}}+\frac{44 b_{1} b_{2} q}{35 z^{2}}\right) x^{10} r_{o s}{ }^{10}+\ldots\right]
\end{aligned}
$$




$$
\begin{aligned}
& F_{4}^{*}=\frac{l(l+1)}{x^{2}}-\frac{l}{x}\left(\frac{d^{2} r_{\psi}}{d x^{2}}\right) /\left(\frac{d r_{\psi}}{d x}\right)+\frac{2 l}{x}\left(\frac{1}{r_{\psi}} \frac{d r_{\psi}}{d x}\right)-\frac{l(l+1)}{r_{\psi}^{2}}\left(\frac{d r_{\psi}}{d x}\right)^{2}, \\
& =-\frac{l}{x^{2}}\left[\frac{4 b_{1}{ }^{2} x^{3} r_{o s}{ }^{3}}{z}+\frac{8 b_{1} b_{2} x^{5} r_{o s}{ }^{5}}{z}+\left(\frac{168 q^{2}}{5 z^{2}}+\frac{56 b_{1}^{2} q}{5 z^{2}}\right) x^{6} r_{o s}{ }^{6}+\frac{448 b_{2}{ }^{2} x^{7} r_{o s}{ }^{7}}{105 z}\right. \\
& +\left(\frac{360 q^{2}}{7 z^{2}}+\frac{576 b_{1} b_{2} q}{21 z^{2}}\right) x^{8} r_{o s}{ }^{8}+\left(\frac{220 q^{2}}{3 z^{2}}+\frac{352 b_{2}{ }^{2} q}{21 z^{2}}\right) x^{10} r_{o s}{ }^{10}+ \\
& l\left\{\frac{2 b_{1}^{2} r_{o s}^{3} x^{3}}{z}+\frac{8 b_{1} b_{2} r_{o s}^{5} x^{5}}{3 z}+\left(\frac{48 q^{2}}{5 z^{2}}+\frac{16 b_{1}{ }^{2} q}{5 z^{2}}\right) x^{6} r_{o s}{ }^{6}+\frac{112}{105} \frac{b_{2}{ }^{2} x^{7} r_{o s}{ }^{7}}{z}+\right. \\
& \left.\left.\left(\frac{80 q^{2}}{7 z^{2}}+\frac{128 b_{1} b_{2} q}{21 z^{2}}\right) x^{8} r_{o s}{ }^{8}+\left(\frac{40 q^{2}}{3 z^{2}}+\frac{8 b_{1} b_{2} q}{7 z^{2}}\right) x^{10} r_{o s}{ }^{10}+\ldots\right\}\right]
\end{aligned}
$$

here $z=M_{0}(r) / M_{1}$ in a binary system $M_{0}$ and $M_{1}$ respectively, are the masses of the primary and secondary components where $\left.\left.M_{0}\right\rangle\right\rangle M_{1}, x=r_{0} / r_{0 s}$ and

$$
\zeta=\frac{r_{\psi}^{2} \delta r_{\psi}}{R^{3} x^{l+1}}, \quad \eta=\frac{\mathrm{P}_{\psi}^{\prime}}{2 \pi \mathrm{G} \rho_{\mathrm{c}} R^{2} x^{l} \rho_{\psi}}, \text { and } \phi=\frac{\psi_{g}^{\prime}}{2 \pi \mathrm{G} \rho_{\mathrm{c}} R^{2} x^{l}}
$$

On setting $z=1, b_{1}^{2}=2 n$, and $b_{2}^{2}=0$ the above expressions reduce the spherical model as each point in the body maintains a constant distance from the rotation axis.

Differential equations (2) analyzing the EF of NRM of pulsations of a (D R) and (T D) stars. Using the boundary conditions (BC) at the free centre and surface it has to be solved. At the centre $\delta r_{\psi}=0, P_{\psi}^{\prime} / \rho_{\psi}=0$ and $\psi_{g}^{\prime}=0$ for $r_{\psi}=0$.

$$
\eta+\phi=\frac{\sigma^{2} \zeta}{2 \pi G \rho_{c} l r_{O S}}, \text { and } \frac{d \phi}{d x}=0
$$

Other demand of free surface $\left(r_{\psi}=R_{\psi}\right)$ is, for $P_{\psi}=0, \delta P_{\psi}=0$. It brings about the situations:

$$
\begin{aligned}
& 2 \pi G \rho_{c} r_{\psi}^{2} \rho_{\psi} \frac{d r_{\psi}}{d x} \eta+R d P_{\Psi} / d x \frac{d P_{\psi}}{d x} \xi=0 \\
& 2 \pi G \rho_{c} \rho_{\psi} R^{2} r_{0 s}^{3}\left[1+\frac{2 b_{1}^{2} x^{3} r_{0 s}^{3}}{z}+\frac{32 b_{1} b_{2} x^{5} r_{0 s}^{5}}{15 z}+\left(\frac{36 q^{2}}{5 z^{2}}+\frac{12 b_{1}^{2} q}{5 z^{2}}\right) x^{6} r_{0 s}^{6}+\right. \\
& \left.\frac{16 b_{2}^{2} x^{7} r_{0 s}^{7}}{21 z}+\left(\frac{55 q^{2}}{7 z^{2}}+\frac{88 b_{1} b_{2} q}{21 z^{2}}\right) x^{8} r_{0 s}^{8}+\left(\frac{26 q^{2}}{3 z^{2}}+\frac{124 b_{1} b_{2} q}{35 z^{2}}\right) x^{10} r_{0 s}^{10}+\ldots\right] \eta+\frac{d P_{\psi}}{d x} \xi=0 .
\end{aligned}
$$

The, B.C gets replaced entirely if the pressure on the free surface is not assumed zero (0). 


$$
\begin{aligned}
& \frac{2 \pi G \rho_{c} R^{2} \rho_{\psi}}{P_{\psi}} \eta+\frac{1}{P_{\psi}} \frac{d P_{\psi}}{d x} \xi \frac{1}{r_{0 s}^{3}}\left[1-\frac{2 b_{1}^{2} x^{3} r_{0 s}^{3}}{z}-\frac{32 b_{1} b_{2} x^{5} r_{0 s}^{5}}{15 z}-\left(\frac{36 q^{2}}{5 z^{2}}+\frac{12 b_{1}^{2} q}{5 z^{2}}\right) x^{6} r_{0 s}^{6}\right. \\
& \left.-\frac{16 b_{2}^{2} x^{7} r_{0 s}^{7}}{21 z}-\left(\frac{55 q^{2}}{7 z^{2}}+\frac{88 b_{1} b_{2} q}{21 z^{2}}\right) x^{8} r_{0 s}^{8}-\left(\frac{26 q^{2}}{3 z^{2}}+\frac{26 b_{1} b_{2} q}{35 z^{2}}\right) x^{10} r_{0 s}^{10}+\ldots\right] \\
& =\left[\sigma^{2} \rho_{\psi}\left(\frac{d P_{\psi}}{d x}\right)^{-1}-4 \pi G \rho_{\psi}^{2}\left(\frac{d P_{\psi}}{d x}\right)^{-1} \frac{D^{2}}{r}\left\{1+\frac{2 b_{1}^{2} x^{3} r_{0 s}^{3}}{3 z}+\frac{16 b_{1} b_{2} x^{5} r_{0 s}^{5}}{15 z}+\left(\frac{4 q^{2}}{z^{2}}+\frac{4 b_{1}^{2} q}{3 z^{2}}\right) x^{6} r_{0 s}^{6}\right.\right. \\
& \left.+\frac{16 b_{2}^{2} x^{7} r_{0 s}^{7}}{35 z}+\left(\frac{5 q^{2}}{z^{2}}+\frac{8 b_{1} b_{2} q}{3 z^{2}}\right) x^{8} r_{o s}^{8}+\left(\frac{6 q^{2}}{z^{2}}+\frac{18 b_{1} b_{2} q}{35 z}\right) x^{10} r_{0 s}^{10}+\ldots\right\} \\
& -\frac{4}{r_{0 s}^{3}}\left\{1-\frac{b_{1}^{2} x^{3} r_{0 s}^{3}}{z}-\frac{4 b_{1} b_{2} x^{5} r_{0 s}^{5}}{5 z}-\left(\frac{12 q^{2}}{5 z^{2}}+\frac{4 b_{1}^{2} q}{5 z^{2}}\right) x^{6} r_{0 s}^{6}\right. \\
& \left.\left.-\frac{8 b_{2}^{2} x^{7} r_{0 s}^{7}}{35 z}-\left(\frac{15 q^{2}}{7 z^{2}}+\frac{8 b_{1} b_{2} q}{7 z^{2}}\right) x^{8} r_{0 s}^{8}-\left(\frac{2 q^{2}}{z^{2}}+\frac{6 b_{1} b_{2} q}{35 z}\right) x^{10} r_{0 s}^{10}+\ldots\right\}\right] \xi \\
& +\frac{2 \pi G \rho_{c} l(l+1)}{\sigma^{2} r_{0 s}^{2}}(\eta+\phi)\left[1-\frac{2 b_{1}^{2} x^{3} r_{0 s}^{3}}{3 z}-\frac{8 b_{1} b_{2} x^{5} r_{0 s}^{5}}{15 z}-\left(\frac{8 q^{2}}{5 z^{2}}+\frac{8 b_{1}^{2} q}{15 z^{2}}\right) x^{6} r_{0 s}^{6}-\frac{16 b_{2}^{2} x^{7} r_{0 s}^{7}}{105 z}\right. \\
& -\left(\frac{10 q^{2}}{7 z^{2}}+\frac{16 b_{1} b_{2} q}{21 z^{2}}\right) x^{8} r_{0 s}^{8}-\left(\frac{4 q^{2}}{3 z^{2}}+\frac{4 b_{1} b_{2} q}{35 z^{2}}\right) x^{10} r_{0 s}^{10} \\
& +2 \pi G \rho_{\psi}\left(\frac{d P_{\psi}}{d x}\right)^{-1}\left[\frac { 2 \rho _ { \psi } D ^ { 2 } } { r _ { 0 s } } \xi \left\{1+\frac{2 b_{1}^{2} x^{3} r_{0 s}^{3}}{3 z}+\frac{16 b_{1} b_{2} x^{5} r_{0 s}^{5}}{15 z}+\left(\frac{4 q^{2}}{z^{2}}+\frac{4 b_{1}^{2} q}{3 z^{2}}\right) x^{6} r_{0 s}^{6}\right.\right. \\
& \left.+\frac{16 b_{2}^{2} x^{7} r_{0 s}^{7}}{35 z}++\left(\frac{5 q^{2}}{z^{2}}+\frac{8 b_{1} b_{2} q}{3 z^{2}}\right) x^{8} r_{0 s}^{8}+\left(\frac{6 q^{2}}{z^{2}}+\frac{18 b_{1} b_{2} q}{35 z^{2}}\right) x^{10} r_{0 s}^{10}+\ldots\right\} \\
& +(l+1) \rho_{c} R^{2} \phi\left\{1+\frac{b_{1}^{2} x^{3} r_{0 s}^{3}}{z}+\frac{2 b_{1}^{2} x^{5} r_{0 s}^{5}}{z}++\left(\frac{24 q^{2}}{5 z^{2}}+\frac{8 b_{1}^{2} q}{5 z^{2}}\right) x^{6} r_{0 s}^{6}+\frac{56 b_{2}^{2} r_{0 s}^{7}}{105 z}\right. \\
& \left.\left.+\left(\frac{40 q^{2}}{7 z^{2}}+\frac{64 b_{1} b_{2} q}{21 z^{2}}\right) x^{8} r_{0 s}^{8}+\left(\frac{20 q^{2}}{3 z^{2}}+\frac{4 b_{1} b_{2} q}{7 z^{2}}\right) x^{10} r_{0 s}^{10}+\ldots\right\}\right]
\end{aligned}
$$

Across the free surface the gravitational potential to be continuous yields:

$\frac{d \phi}{d x}+\left[1+\frac{(l+1)}{r_{\psi}} \frac{d r_{\psi}}{d x}\right] \phi+\frac{2 R \rho_{\psi}}{\rho_{c} r_{\psi}^{2}} \frac{d r_{\psi}}{d x} \xi=0$

i.e. 


$$
\begin{aligned}
& d \phi / d x+\left[( 2 l + 1 ) \left\{1+\frac{b_{1}^{2} x^{3} r_{0 s}^{3}}{z}+\frac{4 b_{1} b_{2} x^{5} r_{0 s}^{5}}{3 z}+\left(\frac{24 q^{2}}{5 z^{2}}+\frac{8 b_{1}^{2} q}{5 z^{2}}\right) x^{6} r_{0 s}^{6}+\right.\right. \\
& \left.\left.+\frac{56 b_{2}^{2} x^{7} r_{0 s}^{7}}{105 z}+\left(\frac{40 q^{2}}{7 z^{2}}+\frac{64 b_{1} b_{2} q}{21 z^{2}}\right) x^{8} r_{0 s}^{8}+\left(\frac{20 q^{2}}{3 z^{2}}+\frac{4 b_{1} b_{2} q}{7 z^{2}}\right) x^{10} r_{0 s}^{10}+\ldots\right\}\right] \phi \\
& +\frac{2 \rho_{\psi}}{\rho_{c} r_{0 s}}\left[1+\frac{2 b_{1}^{2} x^{3} r_{0 s}^{3}}{3 z}+\frac{16 b_{1} b_{2} x^{5} r_{0 s}^{5}}{15 z}+\left(\frac{4 q^{2}}{z^{2}}+\frac{4 b_{1}^{2} q}{3 z^{2}}\right) x^{6} r_{0 s}^{6}+\frac{16 b_{2}^{2} x^{7} r_{0 s}^{7}}{35 z}+\right. \\
& \left.+\left(\frac{5 q^{2}}{z^{2}}+\frac{8 b_{1} b_{2} q}{3 z^{2}}\right) x^{8} r_{0 s}^{8}+\left(\frac{6 q^{2}}{z^{2}}+\frac{18 b_{1} b_{2} q}{35 z^{2}}\right) x^{10} r_{0 s}^{10}+\ldots\right] \xi=0 ;
\end{aligned}
$$

at the surface $x=1$.

\section{EF of Small Adiabatic NRM of Pulsations of Polytropic Stars.}

System of equation (2) with the B.C (3) comprises the eigen-value problem assesses the outcomes of (D R) and (T D) on the EF of NRM of pulsations of stars. By putting the value of $P_{\psi}=P_{c} \theta^{N+1}$ and $\rho_{\psi}=\rho_{c} \theta^{N}$, system of differential equations (2) having NRM, can be revealed as:

$$
\begin{aligned}
& \frac{d \zeta}{d x}+B_{1}^{\circ} \zeta+\left(B_{2}^{\circ}+\frac{B_{3}^{\circ}}{\omega^{2}}\right) \eta+\frac{B_{3}^{\circ}}{\omega^{2}} \phi=0, \\
& \frac{d \eta}{d x}+\left(E_{1}^{\circ} \omega^{2}+E_{2}^{\circ}\right) \zeta+E_{3}^{\circ} \eta+E_{4}^{\circ} \phi+\frac{d \phi}{d x}=0,
\end{aligned}
$$

and

$$
\frac{\mathrm{d}^{2} \phi}{d x^{2}}+F_{1}^{\circ} \frac{d \phi}{d x}+F_{2}^{\circ} \zeta+F_{3}^{\circ} \eta+F_{4}^{\circ} \phi=0
$$

where,

$$
\begin{aligned}
B_{1}^{\circ}= & \frac{l+1}{x}+\frac{N+1}{\gamma}\left(\frac{1}{\theta_{\psi}} \frac{d \theta_{\psi}}{d x}\right), \\
B_{2}{ }^{\circ}= & \frac{(N+1) \xi_{u}{ }^{2} r_{o s}^{3} x}{2 \gamma k^{2} \theta_{\psi}}\left[1+\frac{2 b_{1}{ }^{2} r_{o s}{ }^{3} x^{3}}{z}+\frac{32 b_{1} b_{2} r_{o s}{ }^{5} x^{5}}{15 z}+\left(\frac{36 q^{2}}{5 z^{2}}+\frac{12 b_{1}^{2} q}{5 z^{2}}\right) x^{5} r_{o s}^{5}+\ldots\right. \\
& \left.+\frac{16}{21} \frac{b_{2}{ }^{2} r_{o s}^{7} x^{7}}{z}+\left(\frac{55 q^{2}}{7 z^{2}}+\frac{88 b_{1} b_{2} q}{21 z^{2}}\right) x^{8} r_{o s}{ }^{8}+\left(\frac{26 q^{2}}{3 z^{2}}+\frac{124 b_{1} b_{2} q}{35 z^{2}}\right) x^{10} r_{o s}{ }^{10}+\ldots\right], \\
B_{3}{ }^{\circ}= & -\frac{3 l(l+1) r_{o s}{ }^{4}}{2 k^{3} x}\left(\frac{\rho_{c}}{\rho}\right)\left[1+\frac{4 b_{1}{ }^{2} r_{o s}{ }^{3} x^{3}}{3 z}+\frac{8 b_{1} b_{2} x^{5} r_{o s}{ }^{5}}{5 z}+\left(\frac{28 q^{2}}{5 z^{2}}+\frac{28 b_{1}{ }^{2} q}{15 z^{2}}\right) x^{6} r_{o s}{ }^{6}+.\right. \\
& \left.+\frac{64 b_{2}{ }^{2} x^{7} r_{o s}{ }^{7}}{105 z}+\left(\frac{45 q^{2}}{7 z^{2}}+\frac{24 b_{1} b_{2} q}{7 z^{2}}\right) x^{8} r_{o s}{ }^{8}+\left(\frac{22 q^{2}}{3 z^{2}}+\frac{22 b_{1} b_{2} q}{35 z^{2}}\right) x^{10} r_{o s}{ }^{10}+\ldots\right],
\end{aligned}
$$




$$
\begin{aligned}
& E_{1}^{\circ}=-\frac{2 k^{3}}{3 r_{o s}{ }^{4} x}\left(\frac{\rho^{-}}{\rho_{c}}\right)\left[1+\frac{2 b_{1}{ }^{2} x^{3} r_{o s}{ }^{3}}{3 z}+\frac{16 b_{1} b_{2} x^{5} r_{o s}{ }^{5}}{15 z}+\left(\frac{4 q^{2}}{z^{2}}+\frac{4 b_{1}{ }^{2} q}{3 z^{2}}\right) x^{6} r_{o s}{ }^{6}+\frac{16 b_{2}{ }^{2} x^{7} r_{0 s}{ }^{7}}{35 z}+\right. \\
& \left.+\left(\frac{5 q^{2}}{z^{2}}+\frac{8 b_{1} b_{2} q}{3 z^{2}}\right) x^{8} r_{o s}{ }^{8}+\left(\frac{6 q^{2}}{z^{2}}+\frac{18 b_{1} b_{2} q}{35 z^{2}}\right) x^{10} r_{o s}{ }^{10}+\ldots\right] \text {, } \\
& E_{2}^{\circ}=\frac{2 k^{2}}{\xi_{u}{ }^{2}}\left(N-\frac{N+1}{\gamma}\right) \frac{1}{\theta_{\psi}}\left(\frac{d \theta_{\psi}}{d x}\right)^{2} \frac{1}{r_{o s}{ }^{3} x}\left[1-\frac{2 b_{1}{ }^{2} x^{3} r_{o s}{ }^{3}}{z}-\frac{32 b_{1} b_{2} x^{5} r_{o s}{ }^{5}}{15 z}-\left(\frac{36 q^{2}}{5 z^{2}}+.\right.\right. \\
& \left.\left.+\frac{12 b_{1}^{2} q}{5 z^{2}}\right) x^{6} r_{o s}{ }^{6}-\frac{16}{21 z} b_{2}{ }^{2} x^{7} r_{o s}{ }^{7}-\left(\frac{55 q^{2}}{7 z^{2}}+\frac{88 b_{1} b_{2} q}{21 z^{2}}\right) x^{8} r_{o s}{ }^{8}-\left(\frac{26 q^{2}}{3 z^{2}}+\frac{26 b_{1} b_{2} q}{35 z^{2}}\right) x^{10} r_{0 s}{ }^{10}+\ldots\right] \text {, } \\
& E_{3}^{\circ}=\frac{l}{x}+\left(N-\frac{N+1}{\gamma}\right) \frac{1}{\theta_{\psi}} \frac{d \theta_{\psi}}{d x}, E_{4}^{\circ}=\frac{l}{x} \\
& F_{1}^{\circ}=\frac{l}{x}\left[2(l+1)-\frac{2 b_{1}^{2} x^{3} r_{o s}^{3}}{z}+\frac{16 b_{1} b_{2} x^{5} r_{o s}^{5}}{3 z}+\left(\frac{24 q^{2}}{z^{2}}+\frac{8 b_{1}^{2} q}{z^{2}}\right) x^{6} r_{o s}{ }^{6}+\right. \\
& \left.+\frac{112 b_{2}{ }^{2} x^{7} r_{o s}{ }^{7}}{35 z}+\left(\frac{40 q^{2}}{z^{2}}+\frac{448 b_{1} b_{2} q}{21 z^{2}}\right) x^{8} r_{o}^{8}+\left(\frac{60 q^{2}}{z^{2}}+\frac{36 b_{1} b_{2} q}{7 z^{2}}\right) x^{10} r_{o s}{ }^{10}+\ldots\right] \text {, } \\
& F_{2}^{\circ}=\frac{2}{r_{o s} x}\left(N-\frac{N+1}{\gamma}\right) \theta_{\psi}^{N-1} \frac{d \theta_{\psi}}{d x}\left[1+\frac{2 b_{1}^{2} x^{3} r_{o s}{ }^{3}}{3 z}+\frac{16 b_{1} b_{2} x^{5} r_{o s}{ }^{5}}{15 z}+\left(\frac{4 q^{2}}{z^{2}}+\right.\right. \\
& \left.\left.\left.\frac{4 b_{1}{ }^{2} q}{3 z^{2}}\right) x^{6} r_{o s}{ }^{6}+\frac{16 b_{2}{ }^{2} x^{7} r_{o s}{ }^{7}}{35 z}+\left(\frac{5 q^{2}}{z^{2}}+\frac{8 b_{1} b_{2} q}{3 z^{2}}\right) x^{8} r_{o s}{ }^{8}+\left(\frac{6 q^{2}}{z^{2}}+\frac{18 b_{1} b_{2} q}{35 z^{2}}\right) x^{10} r_{o s}{ }^{10}\right)+\ldots\right] \text {, } \\
& F_{3}^{\circ}=-\frac{(N+1)}{\gamma} \frac{\xi_{u}{ }^{2}}{k^{2}} \theta_{\psi}{ }^{N-1} r_{o s}{ }^{2}\left[1+\frac{8 b_{1}{ }^{2} x^{3} r_{o s}{ }^{3}}{3 z}+\frac{16 b_{1} b_{2} x^{5} r_{o s}{ }^{5}}{5 z}+\left(\frac{56 q^{2}}{5 z^{2}}+\frac{56 b_{1}{ }^{2} q}{15 z^{2}}\right) x^{6} r_{o s}{ }^{6}\right. \\
& \left.+\frac{128 b_{2}{ }^{2} x^{7} r_{o s}{ }^{7}}{105 z}+\left(\frac{90 q^{2}}{7 z^{2}}+\frac{48 b_{1} b_{2} q}{7 z^{2}}\right) x^{8} r_{o s}{ }^{8}+\left(\frac{44 q^{2}}{3 z^{2}}+\frac{44 b_{1} b_{2} q}{35 z^{2}}\right) x^{10} r_{o s}{ }^{10}+\ldots\right] \text {, } \\
& F_{4}^{\circ}=-\frac{1}{x^{2}}\left[l \left\{\frac{4 b_{1}{ }^{2} x^{3} r_{o s}{ }^{3}}{z}+\frac{8 b_{1} b_{2} x^{5} r_{o s}{ }^{5}}{z}+\left(\frac{168 q^{2}}{5 z^{2}}+\frac{56 b_{1}^{2} q}{5 z^{2}}\right) x^{6} r_{o s}{ }^{6}+\frac{448 b_{2}{ }^{2} x^{7} r_{o s}{ }^{7}}{105 z}\right.\right. \\
& \left.+\left(\frac{360 q^{2}}{7 z^{2}}+\frac{576 b_{1} b_{2} q}{21 z^{2}}\right) x^{8} r_{o s}{ }^{8}+\left(\frac{220 q^{2}}{3 z^{2}}+\frac{44 b_{1} b_{2} q}{7 z^{2}}\right) x^{10} r_{o s}{ }^{10}\right\}+l\left\{\frac{2 b_{1}{ }^{2} x^{3} r_{0 s}{ }^{3}}{z}+\right. \\
& +\frac{8 b_{1} b_{2} x^{5} r_{o s}{ }^{5}}{3 z}+\left(\frac{48 q^{2}}{5 z^{2}}+\frac{16 b_{1}{ }^{2} q}{5 z^{2}}\right) x^{6} r_{o s}{ }^{6}+\frac{112}{105} \frac{b_{2}{ }^{2} x^{7} r_{o s}{ }^{7}}{z}+\left(\frac{80 q^{2}}{7 z^{2}}+\frac{128 b_{1} b_{2} q}{21 z^{2}}\right) x^{8} r_{o s}{ }^{8} \\
& \left.\left.+\left(\frac{40 q^{2}}{3 z^{2}}+\frac{8 b_{1} b_{2} q}{7 z^{2}}\right) x^{10} r_{o s}{ }^{10}+\ldots\right\}\right] \text {, }
\end{aligned}
$$

The expressed parameters $\xi_{u}, \rho_{c}$ and $\bar{\rho}$ are equivalent to undistorted polytropic models. B.C (3) in case of deformed polytropic models, at the centre $(x=0)$ is reduced as: 
$\eta+\phi=\frac{2 \omega^{2}}{3 l r_{0 s}^{4}}\left(\frac{\bar{\rho}}{\rho_{c}}\right) \zeta$, and $\frac{d \phi}{d x}=0$

On substituting the values of $P_{\psi}$, and $\rho_{\psi}$ the boundary conditions for free surface $(x=1)$, reduced to;

$$
\begin{aligned}
\eta r_{o s}{ }^{3}[ & 1+\frac{2 b_{1}{ }^{2} x^{3} r_{o s}{ }^{3}}{z}+\frac{32 b_{1} b_{2} x^{5} r_{o s}{ }^{5}}{15 z}+\frac{36 q^{2} x^{6} r_{o s}{ }^{6}}{5 z^{2}}+\frac{16 b_{2}{ }^{2} x^{7} r_{o s}{ }^{7}}{21 z}+\frac{55 q^{2} x^{8} r_{0 o}{ }^{8}}{7 z^{2}}+ \\
& \frac{26 q^{2} x^{10} r_{0 o}{ }^{10}}{3 z^{2}}+2 \frac{k^{2}}{\xi_{u}{ }^{2}} \frac{d \theta_{\psi}}{d x} \zeta=0, \\
\frac{d \phi}{d x}+\phi\left[( l + 1 ) \left\{1+\frac{b_{1}{ }^{2} x^{3} r_{o s}{ }^{3}}{z}+\frac{4 b_{1} b_{2} x^{5} r_{o s}{ }^{5}}{3 z}+\frac{24 q^{2} x^{6} r_{o s}{ }^{6}}{5 z^{2}}+\frac{56 b_{2}{ }^{2} x^{7} r_{o s}{ }^{7}}{105 z}+\right.\right. & \\
+ & \left.\left.\frac{40 q^{2} x^{8} r_{o s}{ }^{8}}{7 z^{2}}+\frac{20 q^{2} x^{10} r_{o s}{ }^{10}}{3 z^{2}} .\right\}+l\right]=0,
\end{aligned}
$$

Differential equations (6) together with the B.C (7-9) calculate the effects of (D R) and (T D) on the EF of NRM of pulsations of polytropic stars. No distortion, (i.e. $z=1, b_{1}=b_{2}=0$ ), differential equations (6) with BC (7-9) is reduced to the usual (eigen-valued) problem determining NRM of pulsations of undistorted polytropic models. This system is reduced to solid body rotation by setting $z=1, b_{1}^{2}=2 \mathrm{n}, b_{2}^{2}=0$ of NRM of pulsations.

\section{Numerical Evaluation}

Using Chebyshev polynomial expansion technique EF of NRM of oscillations of (D R) and (T D) polytropic models have also been calculated and presented by Figure 1 and Figure 2 prior used by Mohan et al. (1991). Symbols are presented in Table 1. Required parameter value of $r_{o s}$ is taken by Lal (1993) as mentioned in Table 2. The essential formulations of the approach given in Saxena (1984). The B.C (8) has been used as the discriminate condition and $\xi=1$ has been used as the regularize condition, at the centre. The utility of $z, \theta_{\psi}$ and $\frac{d \theta_{\psi}}{d x}$ required at various positions in the inner structure of a distorted model, acquired with the resultants of the structure equation for these models, earlier obtained by Lal (1993). For polytropic indices 1.5 and 3.0 generally 10 to 15 collocation points have been used. Whereas, for discovering the EF of higher modes of NR pulsations, the number of collocation points were increased, for getting the required accuracy of .0001 for the same model of indices 1.5 and 3.0 in getting the discriminant condition satisfied. To determine particular mode of NR pulsation of a deformed model the number of collocation points were same as used in undistorted model. Tables 3 and Table 4 represents numerical result for polytropic indices 1.5 and 3.0. The amount of nodes appearing in the eigenfunctions $\xi$ and $\eta$ are also shown in parenthesis in these tables. 
Table 2. Values of $r_{o s}$ and distortion parameters for polyropic indices 1.5 and 3.0.

\begin{tabular}{|c|c|c|c|c|c|}
\hline \multirow{2}{*}{ Stellar Model } & \multicolumn{2}{|c|}{ Values of rotational and tidal distortion parameters } & \multicolumn{2}{c|}{ Polytropic index values of $r_{\text {os }}$} \\
\hline & $\boldsymbol{b}_{\mathbf{1}}$ & $\boldsymbol{b}_{\mathbf{2}}$ & $\boldsymbol{q}$ & $\mathbf{1 . 5}$ & $\mathbf{3 . 0}$ \\
\hline 1. & 0.0000 & 0.0000 & 0.1000 & 0.499815 & 0.499935 \\
\hline 2. & 0.3162 & 0.0000 & 0.1000 & 0.496235 & 0.498620 \\
\hline 3. & 0.0000 & 0.3162 & 0.1000 & 0.499805 & 0.499936 \\
\hline 4. & 0.3162 & 0.3162 & 0.1000 & 0.495895 & 0.498510 \\
\hline 5. & 0.2000 & 0.0200 & 0.1000 & 0.498665 & 0.499500 \\
\hline 6. & 0.1000 & -0.0600 & 0.1500 & 0.499245 & 0.499730 \\
\hline
\end{tabular}

Table 3. Eigen frequencies $\omega^{2}=\left(\frac{r_{O S}^{3} R^{3} \sigma^{2}}{G M_{0}}\right)$ of polytropic models of stars with index 1.5.

\begin{tabular}{|c|c|c|c|c|c|c|c|}
\hline Model No. & $g_{3}$ & $g_{2}$ & $g_{1}$ & $f$ & $p_{1}$ & $p_{2}$ & $p_{3}$ \\
\hline 1. & - & - & - & $\begin{array}{c}2.01669 \\
2.4558 \\
(0-0) \\
\end{array}$ & $\begin{array}{c}10.26798 \\
(10.2812) \\
(1-1) \\
\end{array}$ & $\begin{array}{c}23.46957 \\
(23.4920) \\
(2-2) \\
\end{array}$ & $\begin{array}{c}41.02976 \\
(41.2378) \\
(3-3) \\
\end{array}$ \\
\hline 2. & - & - & - & $\begin{array}{c}2.120807 \\
(2.3856)\end{array}$ & $\begin{array}{c}9.64289 \\
(10.1135)\end{array}$ & $\begin{array}{l}21.08376 \\
(23.1136)\end{array}$ & $\begin{array}{l}36.96368 \\
(40.5845)\end{array}$ \\
\hline 3. & - & - & - & $\begin{array}{l}2.11610 \\
(2.4198)\end{array}$ & $\begin{array}{l}10.26426 \\
(10.2779)\end{array}$ & $\begin{array}{l}23.46076 \\
(23.4854)\end{array}$ & $\begin{array}{l}41.00848 \\
(41.2321)\end{array}$ \\
\hline 4. & - & - & - & 1.99002 & 9.51642 & 21.780768 & 38.3473 \\
\hline 5. & - & - & - & 2.04887 & 9.86694 & 22.5644 & 39.6534 \\
\hline 6. & - & - & - & 2.0773 & 10.00394 & 22.95472 & 40.3310 \\
\hline
\end{tabular}

Table 4. Eigenfrequencies $\omega^{2}=\left(\frac{r_{O S}^{3} R^{3} \sigma^{2}}{G M_{0}}\right)$ of polytropic models of stars with index 3.0.

\begin{tabular}{|c|c|c|c|c|c|c|c|}
\hline Model No. & $\boldsymbol{g}_{\mathbf{3}}$ & $\boldsymbol{g}_{\mathbf{2}}$ & $\boldsymbol{g}_{\mathbf{1}}$ & $\boldsymbol{f}$ & $\boldsymbol{p}_{\mathbf{1}}$ & $\boldsymbol{p}_{\mathbf{2}}$ & $\boldsymbol{p}_{\mathbf{3}}$ \\
\hline & 1.8497 & 2.8296 & 4.8399 & 8.0070 & 14.2672 & 24.6246 & 37.98749 \\
1. & $(1.8700)$ & $(2.8500)$ & $(4.8932)$ & $(8.2487)$ & $(15.2517)$ & $(26.6736)$ & $(41.3569)$ \\
& $(3-3)$ & $(2-2)$ & $(1-1)$ & $(0-0)$ & $(1-1)$ & $(2-2)$ & $(3-3)$ \\
\hline 2. & 1.8296 & 2.8370 & 4.7898 & 7.9408 & 14.4533 & 25.1679 & 38.91084 \\
& $(1.8636)$ & $(2.8400)$ & $(4.8729)$ & $(8.1748)$ & $(15.6271)$ & $(26.2351)$ & $(40.6576)$ \\
\hline 3. & 1.8475 & 2.8383 & 4.8538 & 8.1910 & 15.2319 & 26.6520 & 41.3223 \\
& $(1.8700)$ & $(2.8600)$ & $(4.8936)$ & $(8.2480)$ & $(15.2533)$ & $(26.6655)$ & $(41.3435)$ \\
\hline 4. & 1.8400 & 2.7999 & 4.8130 & 7.9724 & 14.4803 & 25.2145 & 39.0588 \\
\hline 5. & 1.8430 & 2.8199 & 4.8227 & 8.0509 & 14.7805 & 25.7928 & 39.9317 \\
\hline 6. & 1.8565 & 2.8499 & 4.8378 & 8.1158 & 14.9817 & 26.1723 & 40.5416 \\
\hline
\end{tabular}



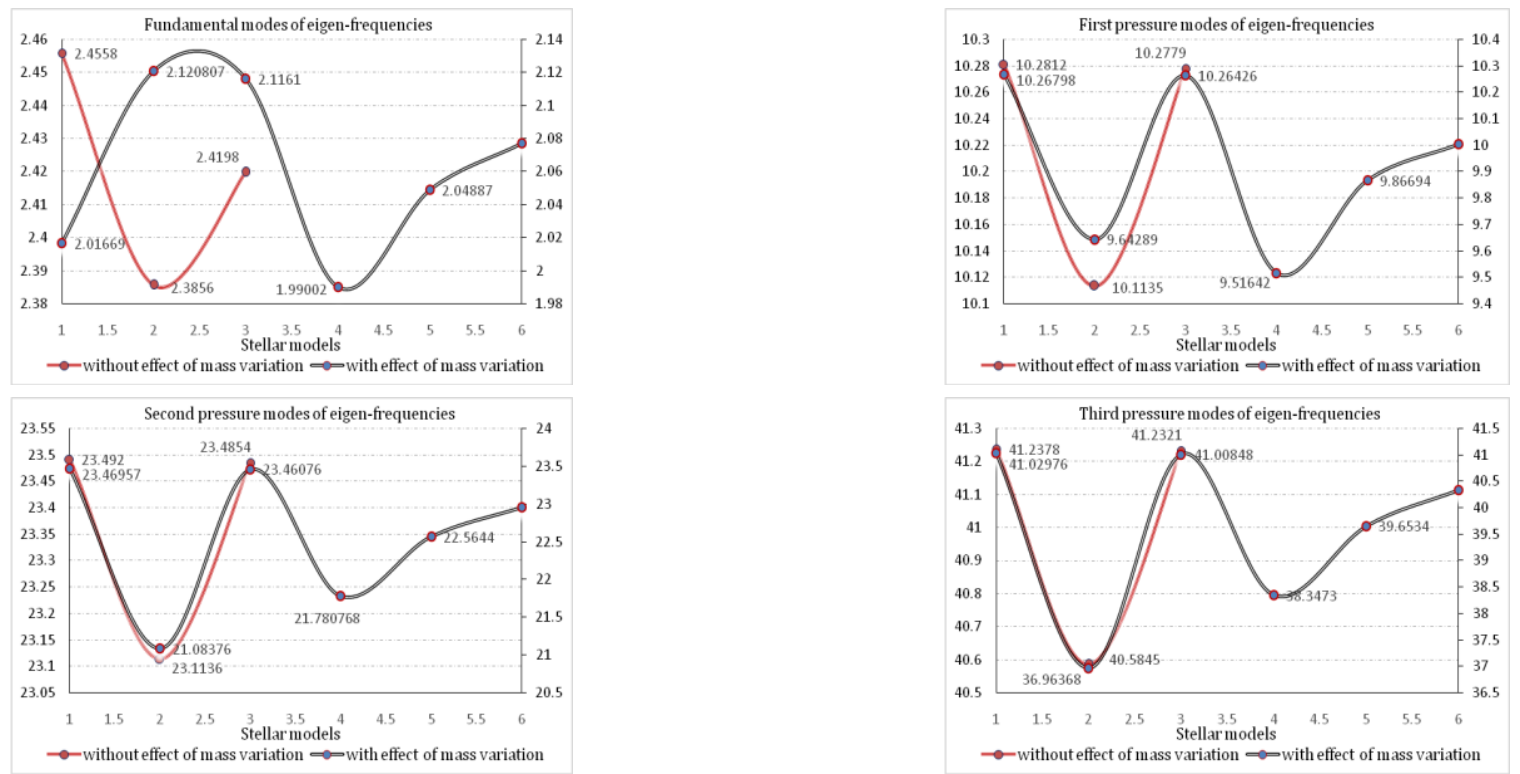

Figure 1. Eigen-frequencies of polytropic stellar models of polytropic index 1.5.
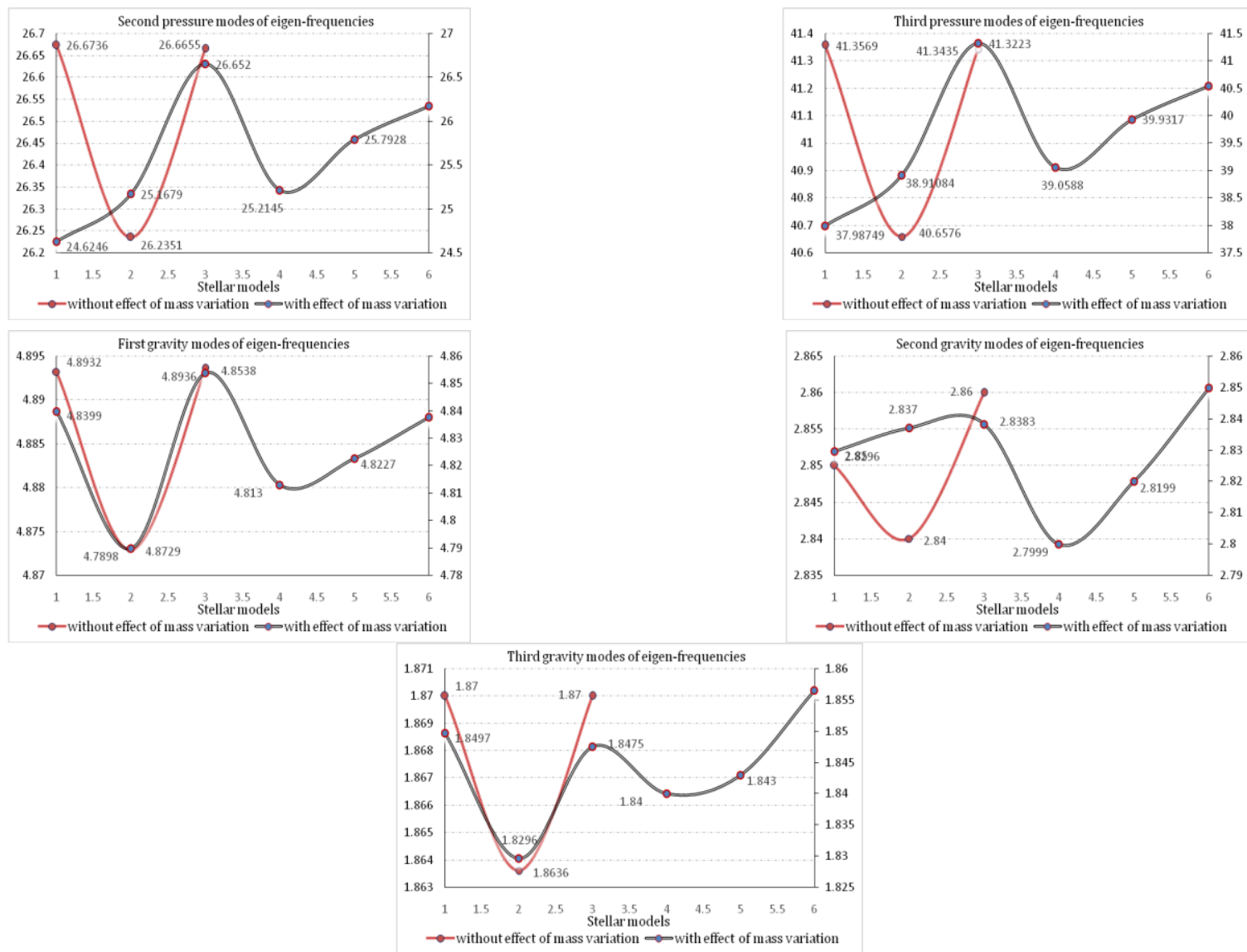

Figure 2. Eigen-frequencies of polytropic stellar models of polytropic index 3.0. 


\section{Result and Discussion}

The eigen-frequencies (EF) of NRM of pulsations of various type of (D R) and (T D) polytropic models with index 1.5 and 3.0 have been presented in Table 3 and Table 4 contains the EF of (gravitational), (fundamental) and (pressure) modes of N-R pulsations of polytropic models of stars. Table 1 shows the presentation of symbols. Table 2 shows the values of $r_{o s}$ for polytropic indices 1.5, 3.0 of (D R) and (T D) models.

On comparing our results for fundamental and pressure mode $\left(f, P_{1}, P_{2}, P_{3}\right)$ with the corresponding results shown in parenthesis earlier obtained by Lal (1993), it is noticed that values of EF obtained by us are smaller. These are also smaller for (D R) and (T D) polytropic models in comparison to tidally distorted polytropic models. The EF of gravitational modes $\left(g_{1}, g_{2}, g_{3}\right)$ of (D R) and (T D) polytropic models 4 and 5 are smaller in comparison to corresponding eigenvalues of tidally distorted polytropic model EF $f, P_{1}, P_{2}, P_{3}$ modes of these models increase. It is also noticed that the EF of $g_{1}, g_{2}, g_{3}$ modes of (D R) and (T D) models increase and those of $f, P_{1}, P_{2}, P_{3}$ modes decrease in comparison to these of tidally distorted polytropic models. However, increment or decrement for these eigenvalues varies for model to model.

\section{Conclusions}

The results based on this study show that the assortment patterns of NR modes of pulsations of the gaseous spheres having mass variation, are similar to the assortment pattern of NR pulsations of same models having constant densities (without mass variation). Pulsars have large EF if they have constant mass structures, observed from these models, but pulsars EF decreased if their structures are considered as spheres of eccentric densities. Enhancement of angular velocity enhances EF of pulsations at the centre of the polytropic stars, while enhancement of angular velocity decreases the pulsations at exterior. The same situation of pulsations can be observed for EF of cosmic models affected by tidally distorted as well as differentially distorted. Addition in tidal effect reduces the EF of (D R) and (T D) stars hence, it can be concluded that inclusion of mass variant affects to NR pulsation of distorted stars. For the future point of view, it will be good to incorporate this approach into certain available computer codes for stellar structure and stellar pulsations and apply it to determine the equilibrium models and discover developmental path of certain real models of distorted pulsars in binary system. Effect of MV on the structures of other type of stellar structures such as white dwarf stars and neutron stars etc. will be observed in our subsequent study.

\section{Conflict of Interest}

The authors confirm that there is no conflict of interest to declare for this publication.

\section{Acknowledgments}

The authors wish to express their appreciation to the Editor and two anonymous reviewers for their valuable comments and recommendations that greatly improve the quality of the manuscript. 


\section{References}

Ball, W.H., Beeck, B., Cameron, R.H., \& Gizon, L. (2016). MESA meets MURaM-surface effects in mainsequence solar-like oscillators computed using three-dimensional radiation hydrodynamics simulations. Astronomy \& Astrophysics, 592(A\&A), A159, 1-8.

Brown, T.M. (1984). Solar p-mode eigenfrequencies are decreased by turbulent convection. Science, 226(4675), 687-689. DOI: 10.1126/science.226.4675.687.

Chandrasekhar, S. (1964). Dynamical instability of gaseous masses approaching the Schwarzschild limit in general relativity. Physical Review Letters, 12(4), 114-116.

Chandrasekhar, S., \& Ferrari, V. (1991). On the non-radial oscillations of a star. Proceedings of the Royal Society of London. Series A: Mathematical and Physical Sciences, 432(1885), 247-279.

Clement, M.J. (1967). Non-radial oscillations and the beta canis majoris stars. II. The effect of differential rotation. The Astrophysical Journal, 150, 589.

De Ridder, J., Barban, C., Baudin, F., Carrier, F., Hatzes, A.P., Hekker, S., Kallinger, T., Weiss, W.W., Baglin, A., Auvergne, M., Samadi, R., Barge, P., \& Deleuil, M. (2009). Non-radial oscillation modes with long lifetimes in giant stars. Nature, 459(7245), 398-400.

Flores, C.V., \& Lugones, G. (2010). Radial oscillations of color superconducting self-bound quark stars. Physical Review D, 82(6), 063006. DOI: 10.1103/PhysRevD.82.063006.

Glendenning, N.K. (2012). Compact stars: nuclear physics, particle physics and general relativity. Springer Science \& Business Media.

Kippenhahn, R., \& Thomas, H.C. (1970). Stellar Rotation, D. Reidel Publ. Co. Dordrecht, Holland, p. 20. In A. Slettebak (ed.).

Kumar, S., Lal, A.K., \& Saini, S. (2015). Vibrational stability of differentially rotating polytropic stars. Astrophysics, 58(1), 120-130. DOI: 10.1007/s10511-015-9370-0.

Lal, A.K. (1993). Structure oscillations and stability of differentially rotating and tidally distorted stellar models (Doctoral dissertation, Ph.D. Thesis, University of Roorkee, Roorkee, India).

Lal, A.K., Pathania, A., Bhalla, A., \& Mohan, C. (2009). Effects of differential rotation on the eigenfrequencies of small adiabatic barotropic modes of oscillations of polytropic models of stars. Journal of Physics A: Mathematical and Theoretical, 42(48), 485212.

Lovekin, C.C., \& Deupree, R.G. (2008). Radial and nonradial oscillation modes in rapidly rotating stars. The Astrophysical Journal, 679(2), 1499-1508.

Mohan, C., \& Saxena, R.M. (1985). Effects of rotation and tidal distortion on the periods of small adiabatic oscillations of the polytropic models of the stars. Astrophysics and Space Science, 113(1), 155-169.

Mohan, C., Saxena, R.M., \& Agarwal, S.R. (1991). Eigenfrequencies of small adiabatic barotropic modes of oscillations of rotationally and tidally-distorted stars. Astrophysics and Space Science, 178(1), 89105. DOI: $10.1007 / \mathrm{BF} 00647117$.

Petrovay, K., Erdélyi, R., \& Thompson, M.J. (2007). The effect of abnormal granulation on acoustic wave travel times and mode frequencies. Solar Physics, 240(2), 197-209.

Saini, S., Kumar, S., \& Lal, A.K. (2015). Effect of mass variation on the radial oscillations of differentially rotating and tidally distorted polytropic stars. Astrophysics, 58(2), 296-307.

Saxena, R.M. (1984). The effects of rotation and tidal distortions on the structure and periods of small oscillations of gaseous spheres (Ph.D. Thesis, University of Roorkee, Roorkee, India).

Schlattl, H., Weiss, A., \& Ludwig, H.G. (1997). A solar model with improved subatmospheric stratification. Astronomy and Astrophysics, 322, 646-652. 
Telting, J.H., \& Schrijvers, C. (1997). Line-profile variations of non-radial adiabatic pulsations of rotating stars. III. On the alleged misidentification of tesseral modes. Astronomy and Astrophysics, 317, 742748.

Trampedach, R., Aarslev, M.J., Houdek, G., Collet, R., Christensen-Dalsgaard, J., Stein, R.F., \& Asplund, M. (2017). The asteroseismic surface effect from a grid of 3D convection simulations-I. Frequency shifts from convective expansion of stellar atmospheres. Monthly Notices of the Royal Astronomical Society: Letters, 466(1), L43-L47.

Yojiosaki, (1986). Nonradial pulsation theory of massive stars. Publication of the Astronomical Society of the Pacific, 98(599), 30-32. 